\title{
Infrared spectroscopy of the merger candidate KIC 9832227
}

\author{
Ya. V. Pavlenko ${ }^{1}$, A. Evans ${ }^{2}$, D. P. K. Banerjee ${ }^{3}$, J. Southworth ${ }^{2}$, M. Shahbandeh ${ }^{4}$, and S. Davis ${ }^{4}$ \\ ${ }^{1}$ Main Astronomical Observatory, Academy of Sciences of the Ukraine, Golosiiv Woods, Kyiv-127 03680, Ukraine \\ e-mail: yp@mao.kiev.ua \\ 2 Astrophysics Group, Keele University, Keele, Staffordshire ST5 5BG, UK \\ 3 Physical Research Laboratory, Navrangpura, Ahmedabad, Gujarat 380009, India \\ ${ }^{4}$ Department of Physics, Florida State University, 77 Chieftain Way, Tallahassee, FL 32306-4350, USA
}

Received 26 January 2018 / Accepted 4 April 2018

\begin{abstract}
Context. It has been predicted that the object KIC 9832227 - a contact binary star - will undergo a merger in $2022.2 \pm 0.7$. We describe the near-infrared (NIR) spectrum of this object as an impetus to obtain pre-merger data.

Aims. We aim to characterise (i) the nature of the individual components of the binary and (ii) the likely circumbinary environment, so that the merger - if and when it occurs - can be interpreted in an informed manner.

Methods. We use infrared (IR) spectroscopy in the wavelength range 0.7-2.5 $\mu \mathrm{m}$, to which we fit model atmospheres to represent the individual stars. We use the binary ephemeris to determine the orbital phase at the time of observation.

Results. We find that the IR spectrum is best fitted by a single component with effective temperature $5920 \mathrm{~K}, \log [g]=4.1$, and solar metallicity, consistent with the fact that the system was observed at conjunction.

Conclusions. The strength of the IR $\mathrm{H}$ lines is consistent with a high value of $\log g$, and the strength of the Ca II triplet indicates the presence of a chromosphere, as might be expected from rapid stellar rotation. The He I absorption we observe likely arises in He excited by coronal activity in a circumstellar envelope, suggesting that the weakness of the Ca II triplet is also likely chromospheric in origin.
\end{abstract}

Key words. circumstellar matter - binaries: close - binaries: eclipsing - stars: individual: KIC 9832227 - infrared: stars

\section{Introduction}

There has recently come to light a new class of eruptive events whose outbursts can be attributed to a stellar merger. These events generally display high luminosity at maximum (Bond et al. 2003) $\left(M_{\mathrm{bol}} \sim-10, M_{v} \lesssim-9\right)$ as evidenced by the detection of such variables in M31 (Bond et al. 2003; Williams et al. 2015) and in other galaxies (Kasliwal 2012; Smith et al. 2016). While the nature of these eruptions was initially unclear (with nova eruptions, planet-swallowing stars, and very late thermal pulses having been suggested) the "best-bet" scenario - based on the behaviour of V1309 Sco - is the merger of two stars.

V1309 Sco is the "Rosetta Stone" of stellar mergers, in that its pre-eruptive behaviour and the subsequent eruption are not only consistent with, but conclusively point to, a stellar merger event in a contact binary. The progenitor had an orbital period of 1.4 days that decreased up to the 2008 eruption (Tylenda et al. 2011). The eruptive event that marked the merger began in 2008 March, reaching peak luminosity $\sim 3 \times 10^{4} L_{\odot}$ (Tylenda et al. 2011). Infrared (IR) observations (McCollum et al. 2014) show that it had an IR excess at least a year before the merger. Pejcha (2014) has shown that V1309 Sco experienced mass loss through the outer Lagrangian point, which eventually obscured the binary. Zhu et al. (2013) note that the presence of a significant amount of dust around V1309 Sco suggests that the ejecta following a stellar merger is an ideal environment for dust formation and growth; they showed that $\sim 5.2 \times 10^{-4} M_{\odot}$ of silicate and iron dust was produced in the merger.

KIC 9832227 was identified by Molnar et al. (2015) as a potential merger candidate, and we present here a near-infrared (NIR) spectrum of this candidate merger object.

\section{KIC 9832227}

KIC 9832227 is both an eclipsing and a contact binary, with an orbital period of 0.458 days and amplitude $\sim 0.2$ mag in the optical. Molnar et al. (2017, hereafter M17) found that the orbital period as determined from Kepler data was significantly shorter than in previous years, and that the period derivative was rapidly decreasing, a sure signature of an impending merger; they estimate the time of merger to be $2022.2 \pm 0.7$. M17 determine that the primary (secondary) star has mass $1.4 M_{\odot}\left(0.32 M_{\odot}\right)$, radius $2.6 R_{\odot}\left(0.78 R_{\odot}\right), T_{\text {eff }}=5800 \mathrm{~K}(5920 \mathrm{~K})$ and $\log g=4.19$ (4.10). There is also some evidence for the presence of a third component in the system, with orbital period $590 \pm 8$ days and $M \sin i=0,11 M_{\odot}$. A detailed discussion of the merger potential of KIC 9832227 is given by M17.

\section{Observations}

\subsection{IRTF observation}

KIC 9832227 was observed with the SpeX spectrograph (Rayner et al. 2003) on the $3 \mathrm{~m}$ NASA Infra-Red Telescope Facility (IRTF), Hawaii, on 2017 July 7.54 UT (MJD 57941.54). SpeX was used in the cross-dispersed mode using a $0.5^{\prime \prime} \times 15^{\prime \prime}$ slit resulting in a spectral coverage $0.77-2.50 \mu \mathrm{m}$, at resolving power $R=\lambda / \delta \lambda=1200$. The total integration time was $717 \mathrm{~s}$. The A0V star HD194354 was used as the telluric standard. The data were reduced and calibrated using the Spextool software (Cushing et al. 2005), and corrections for telluric absorption were performed using the IDL tool Xtellcor (Vacca et al. 2003). The observed spectrum, which was dereddened by $E(B-V)=0.03$ (M17), is shown in Fig. 1. 

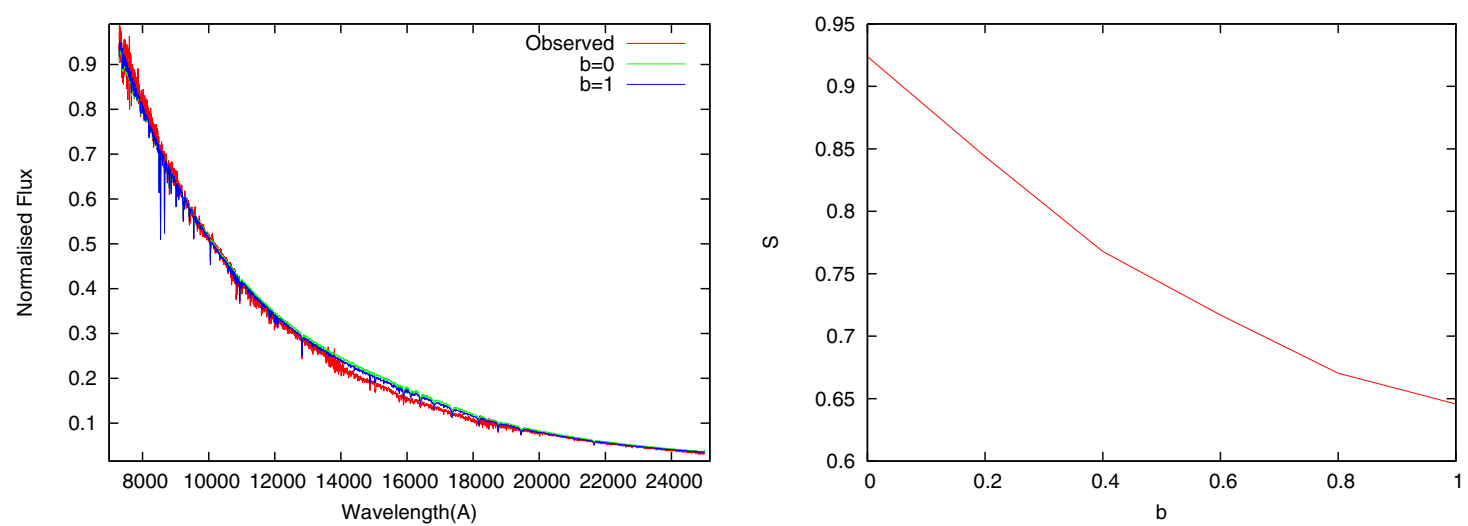

Fig. 1. Left panel: dereddened IRTF spectrum of KIC 9832227, together with the fit with $b=1$; the case $b=0$ is also shown for comparison. See text for details. Right panel: minimisation parameter $S$ as a function of $b$.

Table 1. Infrared fluxes from 2 MASS and WISE.

\begin{tabular}{cccc}
\hline \hline Survey & Band & $\lambda(\mu \mathrm{m})$ & Flux $(\mathrm{mJy})$ \\
\hline \multirow{2}{*}{ MASS } & $J$ & 1.25 & $48.23 \pm 0.92$ \\
& $H$ & 1.65 & $39.55 \pm 0.62$ \\
& $K_{s}$ & 2.2 & $26.59 \pm 0.39$ \\
WISE & $\mathrm{W} 1$ & 3.3 & $13.13 \pm 0.28$ \\
& $\mathrm{~W} 2$ & 4.6 & $7.14 \pm 0.14$ \\
& $\mathrm{~W} 3$ & 12 & $1.31 \pm 0.09$ \\
\hline
\end{tabular}

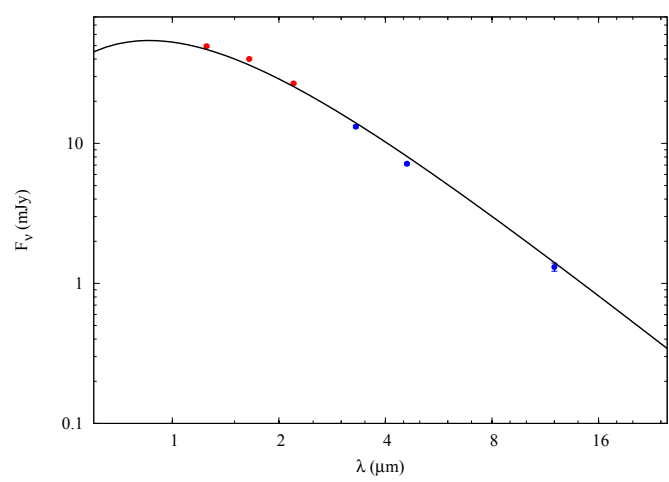

Fig. 2. IR SED of KIC 9832227 from 2 MASS (red) and WISE (blue) surveys. Black curve is a $5930 \mathrm{~K}$ black body, corresponding to the stellar components of KIC 9832227.

\subsection{Complementary observations}

KIC 9832227 is included in the 2MASS (Skrutskie et al. 2006) and WISE (Wright et al. 2010) IR surveys; the source was not detected in other surveys, such as IRAS, AKARI, and Herschel PACS. The 2MASS and WISE fluxes are given in Table 1 and shown in Fig. 2; KIC 9832227 was not detected in WISE Band 4 $(22 \mu \mathrm{m})$. The IR spectral energy distribution (SED) is photospheric out to at least $12 \mu \mathrm{m}$, indicating that dust - if present in significant quantities, as in V1309 Sco - has temperature $\lesssim 425 \mathrm{~K}$.

\subsection{Orbital phase of KIC 9832227 at the time of the IRTF observation}

KIC 9832227 is an eclipsing binary: in order to interpret the IR spectrum, we need to know the orbital phase and stellar velocity separation at the time of the IRTF observation. M17 do not give the full information necessary to deduce these quantities, so this process was non-trivial. We neglected the effect of the third body, which affects the orbital timings by only \pm 0.002 cycles (Fig. 10 in M17), and the proposed three-body solutions suggested by M17 because this body was not detected in the spectrum.

We adopted the parameters of the exponential fit given in Fig. 12 of M17 and the linear orbital ephemeris from the Kepler Eclipsing Binary Catalogue ${ }^{1}$ (see Kirk et al. 2016). We further imposed a normalisation which forces the first point in the year 2004 to be at zero $O-C$. The resulting orbital phases correctly reproduce the $O-C$, instantaneous orbital period, and $\mathrm{d} P / \mathrm{d} t$ as plotted in Figs. 12, 13 and 14 in M17.

The UTC time of mid-observation of our spectrum (2 457 942.04199) was converted into the BJD(TDB) timescale using the IDL routines from Eastman et al. (2010), giving a time of 2457942.04135 . This corresponds to an orbital phase of 0.010 , so our spectrum was obtained around a time of inferior conjunction. The velocity separation of the two stars at this time was negligible compared to their spectral line broadening, so the lines of the two stars are superposed in the spectrum.

\section{Theoretical spectra and SEDs}

Synthetic spectra and SEDs were computed using the WITA6 program for the SAM12 model one-dimensional (1D) atmospheres (Pavlenko 2003) with $T_{\mathrm{e}}($ in $\mathrm{K}) / \log g /[\mathrm{Fe} / \mathrm{H}]=$ $5920 / 4.10 / 0.0$ and 5800/4.19/0.0 for the hotter $(A)$ and cooler (B) components, respectively, as determined by M17. Significant molecular features are not seen in the observed spectrum, therefore we accounted for only atomic lines from the VALD3 database (Ryabchikova et al. 2015). Our procedure for producing the theoretical spectra is described elsewhere (see Pavlenko 2003; Pavlenko et al. 2008). The synthetic spectra were convolved with a Gaussian profile having the instrumental broadening corresponding to the spectral resolution $R$, and rotational profiles corresponding to $v \sin i=85 \mathrm{~km} \mathrm{~s}^{-1}(A)$ and $150 \mathrm{~km} \mathrm{~s}^{-1}$ ( $B$; M17). To account for rotational broadening of the spectra we used formulae given by Gray (1976).

\section{Results}

\subsection{The overall spectrum}

We computed theoretical SEDs for the $A$ and $B$ components, and created a grid of combined spectra $F^{t}=b \times F_{A}+(1-b) \times F_{B}$,

1 http://keplerebs . villanova. edu/overview/?k=9832227 

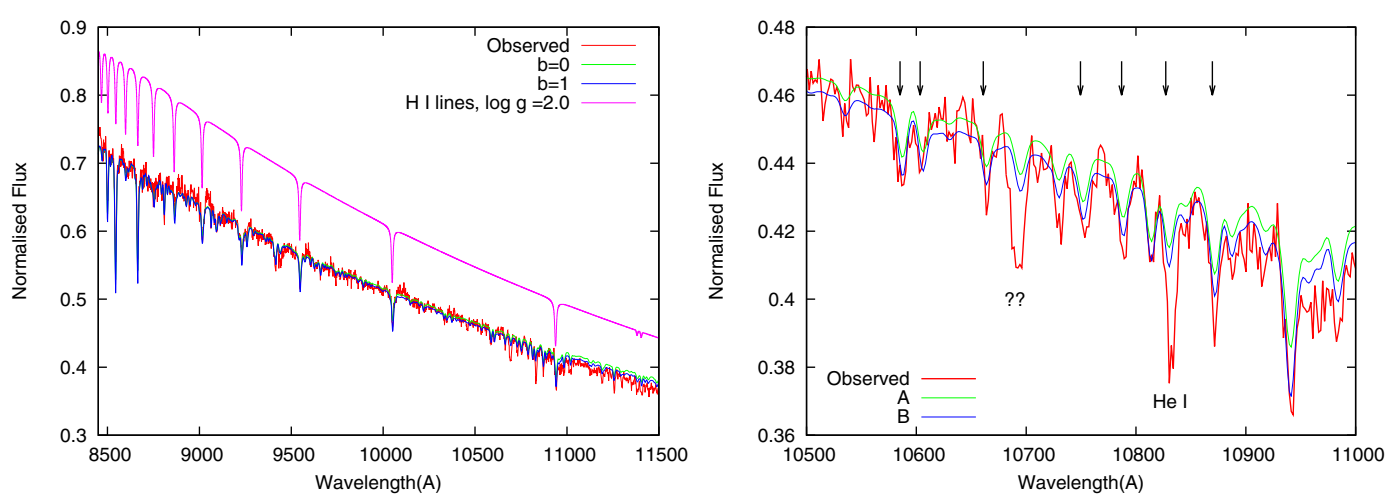

Fig. 3. Left panel: best fit to the observed spectrum with $b=1$ in the region of the Ca II triplet and He I; the case of $b=0$ is also shown, as is the hydrogen line spectrum computed for a model atmosphere with $\log g=2.0$. Right panel: fit to the spectrum containing Si I lines, marked by vertical arrows; the He I line is also shown. We note the broad feature at $10700 \AA$, marked by "??”; see text.

where $0<b<1$. We computed the best fits to the dereddened spectrum for the cases $b=0.0,0.2,0.4,0.6,0.8,1.0$; the procedure to determine the best fit used a $\chi^{2}$ minimising algorithm, details of which are given elsewhere (see Pavlenko et al. 2008). The minimisation parameter $S=\sum\left(F_{\lambda}^{\mathrm{t}}-F_{\lambda}^{\mathrm{obs}}\right)^{2}$ indicates a minimum $S$ at $b=1$ (see Fig. 1). This is consistent with the fact that the observed spectrum was obtained at conjunction, when the hotter component dominates the combined spectrum (although a more compelling reason for not seeing the second component is the very similar $\left.T_{\text {eff }}\right)$. Furthermore, as the eclipses are very shallow, component $A$ dominates the spectrum at all orbital phases.

\subsection{Absorption lines in KIC 9832227's spectrum}

Generally speaking, both the intermediate resolution of the observed spectra $(R=1200)$ and the rapid rotation of both components complicate the use of atomic lines for abundance analysis. However, we see that practically all notable lines in the NIR spectrum are well fitted with close-to-solar abundances; the likely errors do not exceed 0.3 dex. In the right panel of Fig. 3 we show a few well-fitted Si I lines in the spectral range 10 500-11 $000 \AA$. We find fits of the same quality to metallic lines in other spectral regions.

We draw particular attention to the modelling of the moderately strong hydrogen lines, to which our model provides a satisfactory fit. In the left panel of Fig. 3 we show the hydrogen lines computed for the case of lower $\log g=2.0$. In the observed spectrum the upper members of the Brackett series are much weaker; this can be taken as independent evidence of the higher $\log g$, as determined by M17. Indeed, our fits of the theoretical spectra computed for $\log g=4.19$ and $\log g=4.10$ to the observed spectra are far superior.

We also note the presence of a broad feature at $10700 \AA$ (identified by "??" in Fig. 3). Given the width of the feature, a possible identification is $\mathrm{C} I\left({ }^{3} \mathrm{P}-{ }^{2} \mathrm{~S}\right)$ at $10686.01,10688.27$, $10694.17 \AA$; these lines have relatively high excitation potentials $(\sim 7.5 \mathrm{eV})$.

\subsection{The Ca II triplet: evidence of a chromosphere}

Absorption in the Ca II triplet (8 498.023, 8 542.091, $8662.14 \AA$ ) - by comparison with other metallic lines - is a notable feature of KIC 9832227's spectrum (see Fig. 3). This figure shows the relevant portion of Fig. 1 on a larger scale, to compare the observed $\mathrm{Ca}$ II lines with those computed for the case of a classical model atmosphere. The observed lines are notably weaker by comparison with their computed counterparts. The discrepancies between computed and observed Ca II line strengths may be explained by several factors, such as NLTE effects or a Ca deficit. In the former case, NLTE effects in other lines should also be apparent, but this is not the case. Also $\mathrm{HI}$ and metallic lines are well fitted for the case of solar abundances.

The components of the KIC 9832227 system consist of rapidly rotating, tightly bound stars in a contact binary system and we would not expect the atmospheres of such stars so be adequately described by the classical model. In particular, rapid stellar rotation is likely to lead to strong chromospheric (and related) activity, which could certainly account for the weakness of the Ca triplet.

We have simulated a chromosphere by imposing a temperature minimum of $5000 \mathrm{~K}$, and a temperature gradient $G=\mathrm{d} T / \mathrm{d}(\log m)$ ( $m$ being the column density) in the model atmosphere. We fit the Ca II line profiles by choosing the value of $G$ that minimises the fitting parameter $S$ (details of our approach to this calculation are given in Pavlenko 1998). A model with a weak chromosphere $(G=-200$, cf. the solar value $G=-800$, with $T$ in $\mathrm{K}, m$ in $\mathrm{g} \mathrm{cm}^{-2}$ ) provides a good fit to the data in the region of the Ca II triplet (see Fig. 4).

We also note here an alternative interpretation: our classical model atmospheres were computed for Rosseland mean optical depth in the range $\tau_{\text {Ross }}=10^{-5}-100$ but such models lead to Ca II lines that are too strong. Interestingly, a "stripped" model atmosphere for the $A$ component that lacks atmospheric layers in the $\tau_{\text {Ross }}$ range $\left[10^{-5}-3 \times 10^{-2}\right]$ provides an equally good fit to the Ca II triplet (see Fig. 4).

However, while both approaches provide equally good fits to the data, for reasons to be discussed below, we prefer the chromospheric interpretation.

\subsection{He I at $10830 \AA$}

A strong absorption feature is present at $10830 \AA$ (see Fig. 3); we attribute this to He I $10830 \AA\left({ }^{3} \mathrm{~S}-{ }^{3} \mathrm{P}^{0}\right)$, which is also observed in absorption in the spectra of metal-poor subdwarfs (Smith et al. 2012) and red giants (Smith et al. 2014). This line is noticeably narrower than the rotationally broadened photospheric lines, and most likely forms outside the stellar photospheres, possibly in the common envelope.

In the case of the Sun, the line is also observed in absorption; Avrett et al. (1994) suggested that coronal radiation penetrates into the upper solar chromosphere, causing sufficient helium 

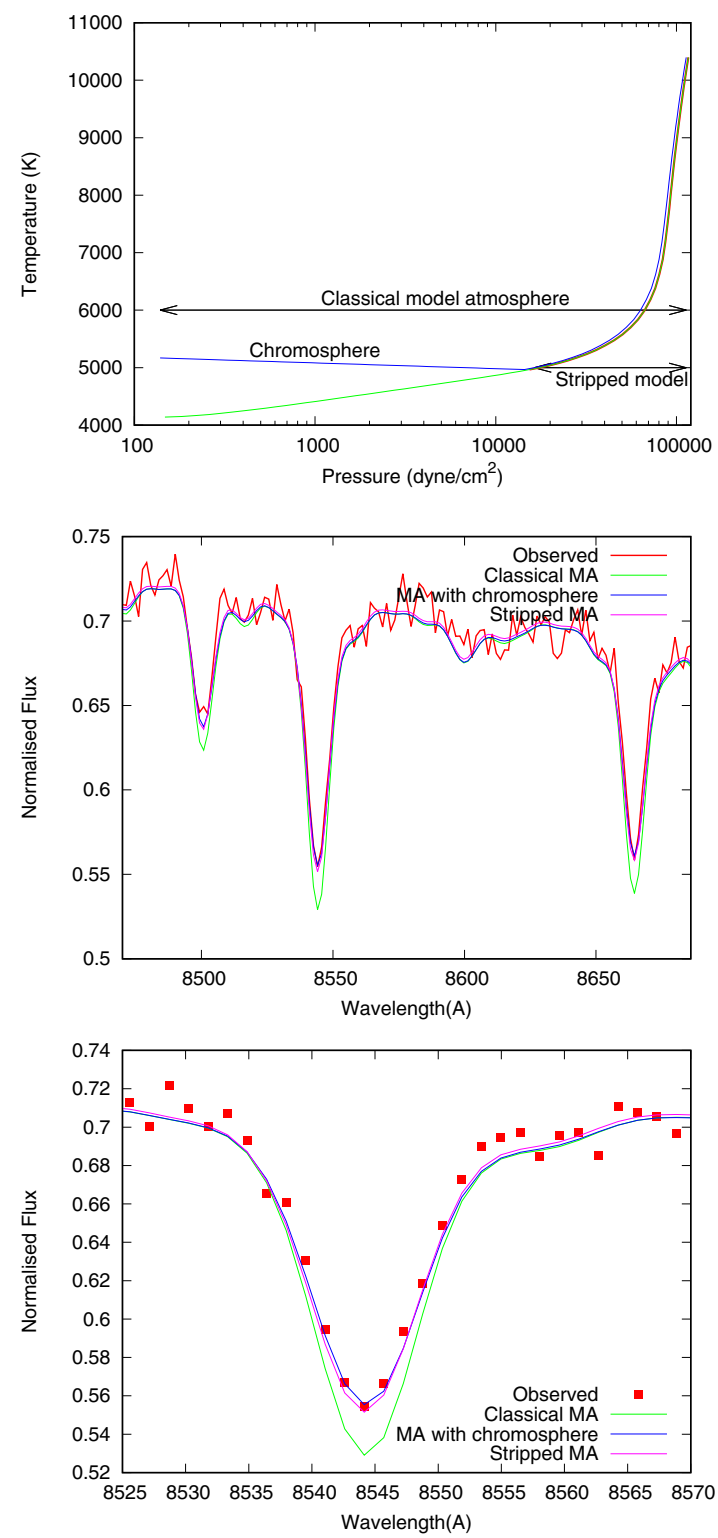

Fig. 4. Top panel: structure of the "classical" and "chromospheric" model atmosphere of component A. Middle panel: best fit to the observed Ca II triplet, with classical and "chromospheric" model atmospheres. Bottom panel: fit to the strongest component of the Ca II triplet shown on a larger scale. In all three panels, the "stripped model atmosphere" refers to the model briefly discussed at the end of Sect. 5.3.

ionisation to populate the lower level of the He I $10830 \AA$ transition, thus resulting in optically thin absorption against the photospheric continuum. This, together with the presence of the C I lines at $\sim 10690 \AA$, lends support to the chromospheric explanation offered in Sect. 5.3.

\section{Conclusions}

We have carried out an analysis of the NIR spectrum of KIC 9832227. We computed synthetic spectra for two classical
1D model atmospheres and fitted the dereddened spectrum. While we acknowledge that the computed model atmospheres may be overly simplistic for the case of the complex KIC 9832227 system, we nonetheless obtain a satisfactory reproduction of the observed SEDs. This may be taken as evidence that, at the time of observation, the 1D+LTE approach is valid.

Within the constraints of our simplified model, we conclude that our ephemeris analysis and spectral synthesis show that, at the time of observation, the contribution of the hotter component prevails in the combined spectrum. The strength of the $\mathrm{HI}$ lines is well reproduced by our models, providing independent confirmation of the high $\log g$ (4.10, component $A ; 4.19$, component $B$; M17) at least in the atmosphere of component $A$; model atmospheres with lower $\log g$ result in Brackett and Paschen lines that are too srong. A comparatively narrow feature at $10830 \AA$ is identified as the He I $\left({ }^{3} \mathrm{~S}-{ }^{3} \mathrm{P}\right)$ line, and is formed in the outer part of the common envelope. A satisfactory fit to the Ca II IR triplet is obtained by introducing a chromosphere into the model atmosphere; this is likely a consequence of rapid stellar rotation. Despite the intermediate resolution of observed spectra and the fast rotation of both components, we confirm that component $A$ has near-solar abundances.

Acknowledgements. We warmly thank David Sand for making possible the IRTF observations, and for help with the data reduction. The Infrared Telescope Facility is operated by the University of Hawaii under contract NNH14CK55B with the National Aeronautics and Space administration. We thank the referee for their helpful comments.

\section{References}

Avrett, E. H., Fontenla, J. M., \& Loeser, R. 1994, in Infrared Solar Physics, eds. D. M. Rabin, J. T. Jefferies, \& C. Lindsey (Dordrecht: Kluwer Academic Publishers), IAU Symp., 154, 35

Bond, H. E., Henden, A., Levay, Z. G., et al. 2003, Nature, 422, 405

Cushing M. C., Rayner J. T., \& Vacca W. D. 2005, ApJ, 623, 1115

Eastman, J., Siverd, R., \& Gaudi, B. S. 2010, PASP, 122, 935

Gray, D. F. 1976, The Observation and Analysis of Stellar Photospheres (New York: Wiley Interscience)

Kasliwal, M. M. 2012, PASA, 29, 482

Kirk, B., Conroy, K. E., Prša, A., et al. 2016, AJ, 151, 68

McCollum, B., Laine, S., Väisänen, P., et al. 2014, AJ, 147, 11

Molnar, L. A., Van Noord, D. M., Steenwyk, S. D., Spedden, C. J., \& Kinemuchi, K. 2015, BAAS, 225, 415.05

Molnar, L. A., Van Noord, D. M., Kinemuchi, K., et al. 2017, ApJ, 840, 1

Nicholls, C. P., Melis, C., Soszyński, I., et al. 2013, MNRAS, 431, L33

Pavlenko, Ya. V. 1998, Astron. Rep., 42, 501

Pavlenko, Ya. V. 2003, Astron. Rep., 47, 59

Pavlenko, Ya. V., Evans, A., Kerr, T., et al. 2008, A\&A, 485, 541

Pejcha, O. 2014, ApJ, 788, 22

Rayner J. T., Toomey D. W., Onaka P. M., et al. 2003, PASP, 115, 389

Ryabchikova, T., Piskunov, N., Kurucz, R. L., et al. 2015, Phys. Scr., 90, 054005

Smith, G. H., Dupree, A. K., \& Strader, J. 2012, PASP, 124, 1252

Smith, G. H., Dupree, A. K., \& Strader, J. 2014, PASP, 126, 901

Smith, N., Andrews, J. E., Van Dyk, S. D., et al. 2016, MNRAS, 458, 950

Strutskie, M. F., Cutri, R. M., Stiening, R. et al. 2006, AJ, 131, 1163

Tylenda, R., Hajduk, M., Kamiński, T., et al. 2011, A\&A, 528, A114

Vacca, W. D., Cushing, M. C., \& Rayner, J. T. 2003, PASP, 115, 389

Williams, S. C., Darnley, M. J., Bode, M. F., \& Steele, I. A. 2015, ApJ, 805, L18

Wright, E. L., Eisenhardt, P. R. M., Mainzer, A. K., et al. 2010, AJ, 140, 1868

Zhu, C., Lü, G., \& Wang, Z. 2013, ApJ, 777, 23 